\title{
Motor Interference, But Not Sensory Interference, Increases Midfrontal Theta Activity and Brain Synchronization during Reactive Control
}

\author{
${ }^{(1) J a k o b ~ K a i s e r ~ a n d ~ S i m o n e ~ S c h u ̈ t z-B o s b a c h ~}$ \\ General and Experimental Psychology, Ludwig-Maximilian-University, D-80802 Munich, Germany
}

Cognitive control helps us to overcome task interference in challenging situations. Resolving conflicts because of interfering influences is believed to rely on midfrontal theta oscillations. However, different sources of interference necessitate different types of control. Attentional control is needed to suppress salient distractors. Motor control is needed to suppress goal-incompatible action impulses. While previous studies mostly studied the additive effects of attentional and motor conflicts, we independently manipulated the need for attentional control (via visual distractors) and motor control (via unexpected response deviations) in an EEG study with male and female humans. We sought to find out whether these different types of control rely on the same midfrontal oscillatory mechanisms. Motor conflicts, but not attentional conflicts, elicited increases in midfrontal theta power during conflict resolution. Independent of the type of conflict, theta power was predictive of motor slowing. Connectivity analysis via phase-based synchronization indicated a widespread increase interbrain connectivity for motor conflicts, but a midfrontal-to-posterior decrease in connectivity for attentional conflicts. For each condition, we found stronger midfrontal connectivity with the parietal region contralateral to, rather than ipsilateral to, the acting hand. Parietal lateralization in connectivity was strongest for motor conflicts. Previous studies suggested that midfrontal theta oscillations might represent a general control mechanism, which aids conflict resolution independent of the conflict domain. In contrast, our results show that oscillatory theta dynamics during reactive control mostly reflect motor-related adjustments.

Key words: cognitive control; distraction; motor control; neural oscillations

\section{Significance Statement}

Humans need to exercise self-control over both their attention (to avoid distraction) and their motor activity (to suppress inappropriate action impulses). Midfrontal theta oscillations have been assumed to indicate a general control mechanism, which help to exert top-down control during both motor and sensory interference. We are using a novel approach for the independent manipulation of attentional and motor control to show that increases in midfrontal theta power and brainwide connectivity are linked to the top-down adjustments of motor responses, not sensory interference. These findings clarify the function of midfrontal theta dynamics as a key aspect of neural top-down control and help to dissociate domain-general from motor-specific aspects of self-control.

\section{Introduction}

Cognitive control helps to maintain goal-directed behavior when we are confronted with internal or external interference. Processing conflicts because of task interference can occur in different domains. Attentional conflicts typically arise because of

Received June 30, 2020; revised Nov. 17, 2020; accepted Dec. 17, 2020.

Author contributions: J.K. and S.S.-B. designed research; J.K. performed research; J.K. analyzed data; J.K. and S.S.-B. wrote the paper.

This work was supported by an LMUexcellent Grant to S.S.-B. We thank Bettina Schmieder, Claudia Werner, Konstantin Steinmassl, and Clara Dominke for help with data collection.

The authors declare no competing financial interests.

Correspondence should be addressed to Jakob Kaiser at J.Kaiser@psy.Imu.de.

https://doi.org/10.1523/JNEUROSCl.1682-20.2020

Copyright $\odot 2021$ the authors the simultaneous presence of task-relevant stimuli and salient distractors, such as when drivers need to focus on the traffic despite colorful advertisements on the roadside (Sawaki and Luck, 2010; Gaspar and McDonald, 2014; Kerzel et al., 2018; Chelazzi et al., 2019). Motor conflicts occur when we must deviate from a prepotent response pattern, such as when drivers need to hit the brakes because of an unexpected stop sign (Botvinick et al., 2004; Egner, 2008; Aron, 2011; Cohen, 2014a). Both attentional and motor conflicts lead to increased error rates and slower reaction times (Criaud and Boulinguez, 2013; Gaspelin and Luck, 2018a; Wessel, 2018; Liesefeld et al., 2019). To understand how the brain enables top-down control of our behavior, it is crucial to find out whether different types of conflicts are resolved by separate, domain-specific or shared, domain-general top-down 
Singleton Distractor Absent

(No Attentional Conflict)

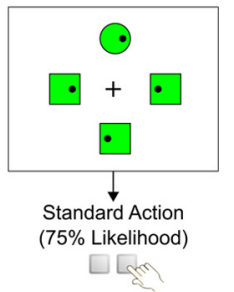

Singleton Distractor Present (Attentional Conflict)

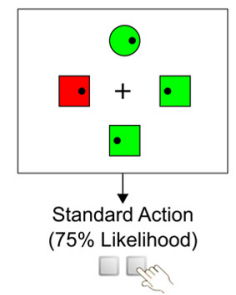

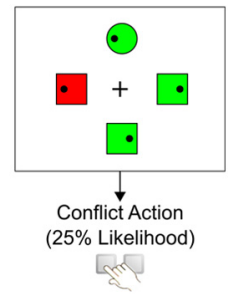

Figure 1. Schematic representation of study design. Figure shows examples for stimulus displays, either with or without salient singleton distractor (red square). Participants had to locate the unique shape (circle) and respond according to the position of the dot within the target shape.

control mechanisms in the brain (Cocchi et al., 2013; Mackie et al., 2013; Hampshire and Sharp, 2015; Gratton et al., 2018).

Motor conflicts lead to an increase in oscillatory power in the theta range $(4-7 \mathrm{~Hz})$ over the midfrontal cortex (Nigbur et al., 2011; Mückschel et al., 2017; Vissers et al., 2018; Kaiser et al., 2019; Kaiser and Schütz-Bosbach, 2019). Higher theta power has been found to predict motor slowing, which is believed to prevent a premature elicitation of prepotent but goal-incompatible impulses (Wessel and Aron, 2013, 2014; Chang et al., 2017; Töllner et al., 2017). Conflict-related increases in midfrontal theta power have been observed in a wide range of different experimental paradigms (Cohen and Cavanagh, 2011; Derosiere et al., 2018; Vissers et al., 2018; Cooper et al., 2019). Therefore, it has been suggested that midfrontal theta oscillations might be indicative of a general control mechanism (Cavanagh and Frank, 2014; Cohen, 2014a). It has been speculated that theta synchronization between midfrontal and other domain-specific brain areas could facilitate the adjustments necessary to overcome different types of conflicts (van Driel et al., 2012; Cooper et al., 2015; Duprez et al., 2019). Cognitive conflicts also often lead to decreases in alpha power (8-14 Hz; e.g., Popov et al., 2018). Lower alpha power has been found to indicate increased attention toward task-relevant stimuli (Clayton et al., 2018; Van Diepen et al., 2019).

For the simultaneous experimental manipulation of attentional and motor conflicts, most previous studies used a version of the flanker paradigm (Verbruggen et al., 2006; Nigbur et al., 2011, 2012; Soutschek et al., 2013; Pastötter and Frings, 2018). Here, different types of target symbols (e.g., letters or numbers) necessitate different motor responses. Each target is accompanied by a congruent or incongruent flanker. Congruent flankers are identical to the target, which poses no interference. Incongruent flankers are either different symbols associated with the target response, which primarily evokes sensory interference, or different symbols associated with a target-incompatible response, evoking both sensory and motor interference. Thus, attentional and motor interference in these studies are necessarily additive. However, for a clear dissociation of attentional and motor control, it is necessary to ensure an independent manipulation of distractor and motor conflicts within the same task. Therefore, the current study combined the singleton-distractor task, where a distractor captures attention because of its unique color (Gaspar and McDonald, 2014), with a Go/Change-Go task, where participants have to change prepotent response patterns (Kaiser and Schütz-Bosbach, 2019). We used this paradigm to compare the effect of attentional and motor conflicts on midfrontal theta power as well as theta synchronization as a measure of interbrain connectivity. This

allowed us to test whether theta oscillations represent a domain-general or task-specific mechanism of top-down control, and in how far the pattern of theta connectivity differs between attentional and motor conflicts.

\section{Materials and Methods}

Participants. Thirty-seven participants (11 male) took part in the experiment for course credits or financial reimbursement of $9 € / \mathrm{h}$. The mean age of participants was 25.0 years $(\mathrm{SD}, 4.1)$. Three datasets were excluded from the analysis, either because they retained $<50$ trials for EEG analysis in at least one experimental condition after data preprocessing $(n=1)$, or because of extreme values in their behavioral performance $(n=2$, see below), resulting in a final sample of 34 participants.

Measurement setup. For recording EEG, we used 65 active electrodes (ActiSnap, BrainProducts) and one additional ground electrode. Electrodes positions followed the international 10-20 system. The $\mathrm{FCz}$ functioned as the online reference. EEG was recorded with a QuickAmp amplifier (BrainVision), using a $500 \mathrm{~Hz}$ sampling rate and a $0.016-250 \mathrm{~Hz}$ online bandpass filter.

Experimental design. Figure 1 shows a schematic overview of the experimental design. On each trial, a search display was shown, which consisted of four items arranged around a fixation cross (size: $0.8^{\circ} \times 0.8^{\circ}$ visual angle) in the middle of the screen. Participants were instructed to keep their eyes on the fixation cross at all times. Each of the items $\left(1^{\circ} \times\right.$ $1^{\circ}$ ) had a distance of $2^{\circ}$ visual angle to the fixation cross. The four stimuli were either three circles and one square or three squares and one circle. The item with the unique shape on each trial was defined as the target. All stimuli contained small black dots $\left(0.1^{\circ} \times 0.1^{\circ}\right)$, which were positioned either on the left or right side of each shape. Participants were instructed to locate the target as quickly as possible and press the left or right arrow button on the keyboard depending on the position of the black dot within the target. All responses had to be performed with the right index finger. The search display remained on the screen for $1.2 \mathrm{~s}$. If participants did not answer within this time period or if they pressed the wrong button, a clock symbol or an X was shown as negative feedback for $0.3 \mathrm{~s}$. The intertrial interval randomly varied between 1.5 and $1.8 \mathrm{~s}$.

To manipulate the degree of attentional conflict, we varied the colors of the search items. On half of the trials, all items had the same color (either green or red, no-distractor trials). On the other half of the trials, one of the nontarget items had a different color than all other items (singleton-distractor trials). Thus, a singleton-distractor trial could consist of either three green items and one red color singleton or three red items with one green color singleton. We hypothesized that singleton-distractor trials would lead to involuntary attentional capture for the nontarget with the unique color, and therefore necessitate increased attentional control. Note that the colors of the items did not have any direct relevance for participants' task. Participants were instructed to always ignore colors, but only look for the target with the unique shape. The assignment of the two colors red/green between singleton-distractor and nonsingleton items varied randomly between trials.

To manipulate the degree of motor conflict, we varied the relative frequency of the two target actions. For each participant, either the left or right button press was prompted in $75 \%$ of all trials (standard action), while the opposite button press was needed in $25 \%$ of the trials (conflict action). It was assumed that prompting the conflict action would increase the need for motor control, since this action deviated from the more frequent standard response. The assignment between left/right and standard/conflict action was counterbalanced between participants.

To ensure that our findings were not unduly influenced by systematic differences between the search displays, we counterbalanced and randomized several factors between trials. Target and singleton distractor appeared equally often in each of the four possible positions around the fixation cross. The positions of the dots in the four items were randomized so that each display contained two left-facing dots and two right-facing dots. For half of the singleton-distractor trials, the distractor 
shape contained a dot that was target compatible, meaning it pointed in the same direction as the dot in the target shape (Fig. 1). For the other half of the trials, the dot in the singleton distractor was target incompatible, meaning that it pointed in the opposite direction of the target (e.g., distractor with left-facing dot, but target with right-facing dot). We included both target-compatible and target-incompatible distractor trials, because in this way we ensured that participants could not infer the target action from merely looking at the distractor, meaning that the salient distractor had no information value for choosing the correct response.

The colors of singleton-distractor and nonsingleton items were counterbalanced, so that in each condition half of the trial used the same target/distractor colors as in the directly preceding trial (e.g., standard items, green; distractor items, red), while the other half of the trials used the opposite color assignment (e.g., standard items, red; distractor items, green). The shapes of targets and nontargets (either circle or square) were randomized across trials.

The experiment consisted of 1920 trials, subdivided into 16 blocks. The order of all trials was randomized. After each block, participants received feedback about their average error rates and reaction times. Breaks between blocks were self-paced. Overall, the experimental procedure took $\sim 90 \mathrm{~min} /$ participant.

Behavioral analysis. For both behavioral and EEG analysis, we retained only trials with correct responses, leading to the mean removal of $4.93 \%$ (SD, 2.52\%) of all trials. For trials with salient distractors, we included in the main analysis only target-compatible distractors, where the distractor dot was located at the same side as the target dot (Fig. 1). This was done because distractors associated with target-incompatible actions are known to elicit motor conflicts, and therefore would have confounded attentional and motor interference (Verbruggen et al., 2006).

Error rates and reaction times for correct responses were averaged for each condition. Box plots were used for the identification of outliers. More specifically, we excluded all participants whose error rates or reaction times deviated $>1.5$ times of the interquartile range from the overall sample (Schwertman et al., 2004). This led to the exclusion of two participants. Both behavioral measures were analyzed with repeated-measures ANOVAs with the factors ATTENTION (no attentional conflict/attentional conflict) and MOTOR (no motor conflict/motor conflict). As effect size, we report $\eta_{p}^{2}$ and Cohen's $d$. Additionally, we report Bayes factors $(\mathrm{BFs})$ that indicate the evidence for the alternative hypothesis $(\mathrm{BF}>1)$ relative to evidence for the null hypothesis $(\mathrm{BF}<1)$. It has been suggested that $\mathrm{BF}>10$ represents strong evidence for the alternative hypothesis, while $\mathrm{BF}<0.10$ indicates strong evidence for the null hypothesis (Jarosz and Wiley, 2014; Van de Schoot et al., 2014; Wagenmakers et al., 2018). Tests were calculated with the $\mathrm{R}$ packages ez, effsize, and BayesFactor.

EEG preprocessing. EEG preprocessing and data analysis was performed with the MATLAB Toolbox Fieldtrip (Oostenveld et al., 2011). For five participants, one to four exceedingly noisy electrodes were excluded and later replaced via interpolation using the function $f t$ channelrepair. EEG data were referenced to an average of all electrodes and filtered (low-pass filter, $100 \mathrm{~Hz}$; high-pass filter, $0.75 \mathrm{~Hz}$ ). To facilitate further processing, data were downsampled to $250 \mathrm{~Hz}$. We then extracted for each trial the EEG activity between -1.5 and $+2.2 \mathrm{~s}$ around trial onset. This time interval was chosen to be considerably longer than the overall trial length, to avoid edge effects during the time-frequency calculation. We used independent component analysis to identify components that represented eye blinks or other non-brain-related artifacts. This led to the removal of one to three components per participant (mean, 1.53). Furthermore, we removed all trials where any EEG channel showed deflections higher than $90 \mu \mathrm{V}$, resulting in an average exclusion rate of $6.56 \%(\mathrm{SD}, 5.25)$ of all trials. High voltage deflections are most likely to stem from noise artifacts, such as strong body movements, and are therefore commonly removed from EEG datasets (Yamanaka and Yamamoto, 2010; Gaspelin and Luck, 2018a,b; Cooper et al., 2019). To ensure a sufficient number of trials in each condition, we only retained participants with at least 50 trials per condition, leading to one exclusion. For the final sample, we retained an average of 638.0 trials for each participant (minimum, 546; maximum, 717) without conflict, 325.0 trials (range, 265-357 trials) with only attentional conflicts, 210.5 trials (range, 171-236 trials) with only motor conflicts, and 100.3 trials (range, 77-115 trials) with both attentional and motor conflicts. Resulting datasets were filtered with the function $f t$ scalpcurrentdensity using a Laplacian (spatial) filter with the spline method and a polynomial degree of 10 . This increases the spatial specificity for the results of time-frequency and connectivity analysis (Cohen, 2015).

Statistical analysis. Time-frequency data were calculated using Morlet wavelets, as implemented in the function ft_freqanalysis. For each condition, we estimated the oscillatory power from 1 to $20 \mathrm{~Hz}$ in 1 $\mathrm{Hz}$ steps, while increasing the number of wavelet cycles from three to eight cycles in linearly spaced steps. These processing parameters are in line with general recommendations and previous studies concerning neural theta oscillations (Cohen, 2014b; Cooper et al., 2016; Harper et al., 2017).

The main goal of our analysis was to compare the neural impact of attentional conflicts evoked by distractor displays with motor conflicts evoked by deviations from the prepotent motor response. If attentional control was associated with midfrontal theta oscillations, we would expect that the onset of distractor displays elicits a change in midfrontal theta activity. Thus, a stimulus-locked analysis relative to the distractor onset should identify neural processes elicited by sensory distraction. Concerning motor control, we would expect that motor conflicts lead to an increase in midfrontal theta power before action execution. However, since both attentional and motor conflicts have been shown to slow down reaction times, stimulus-locked differences in activity between conditions around the time of the motor response could simply reflect slower reactions during conflict trials (Gaspelin and Luck, 2018a; Wessel, 2018). Thus, a response-locked analysis relative to the motor response is more appropriate for comparing processes related to response preparation across different conditions. Accordingly, we extracted time-frequency data in the following two different ways: stimulus centered, defined as $0-1.2 \mathrm{~s}$ relative to the onset of the search display; and response centered, defined as -0.8 to $0.4 \mathrm{~s}$ around the motor response based on reaction times on each trial. Where appropriate, we present the results of both stimulus-centered and response-centered analyses to aid the distinction between distractor-evoked and responselocked neural processes. For each condition, we calculated average power values. Condition-wise averages were baseline corrected via decibel conversion, using the mean power of all trials between 0.3 and $0.1 \mathrm{~s}$ before trial onset as baseline value. Using a trial average as the baseline for the calculation of time-frequency maps increases the signal-to-noise ratio (Cohen, 2014b).

Statistical analysis of time-frequency maps was based on the average of midfrontal electrodes FCz, FC1, and FC2. These electrodes were chosen, because previous studies of midfrontal theta power overwhelmingly found the peak of theta power around this site (Cohen and Cavanagh, 2011; Chang et al., 2017; Vissers et al., 2018; Kaiser and Schütz-Bosbach, 2019). Topographical plots of our data confirmed that this location represented the peak of neural theta increases (Fig. 2). We used cluster-based permutation analysis to identify significant effects of the main factors ATTENTION, MOTOR, and the ATTENTION * MOTOR interaction in the time-frequency data (Derosiere et al., 2018). Permutation analysis allows for statistical tests over whole timefrequency maps, while still controlling for multiple comparisons (Maris and Oostenveld, 2007). More specifically, for each main factor and the interaction separately, statistical contrasts were first determined via $F$ tests for each individual time-frequency point. Adjacent data points below a cutoff of $p<0.05$ were combined into clusters. The weight of each cluster was calculated as the sum of the $F$ values of all the tests for its individual time points. The statistical significance of each cluster was calculated as the probability that a cluster with equal weight could appear because of chance, as determined via 10,000 random permutations over the original data. We report all clusters that were significant with $p<0.05$. We also calculated post hoc contrasts between individual conflict conditions, using the same permutation approach, but, instead of $F$ tests, we used $t$ tests, which were corrected for two-sided comparisons. 

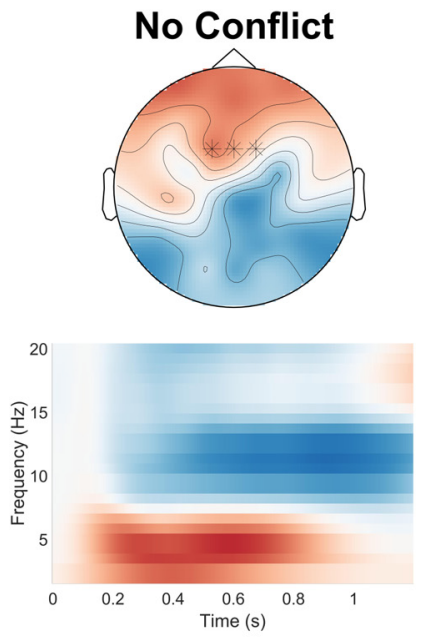

Attentional Conflict
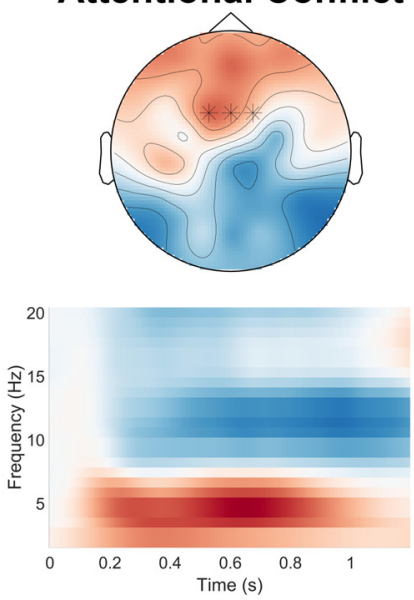

Motor Conflict
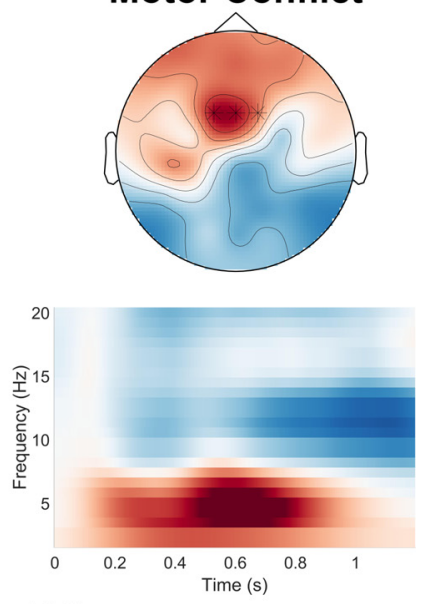

Power (dB)

$\begin{array}{lll}-2 & 0 & 2\end{array}$

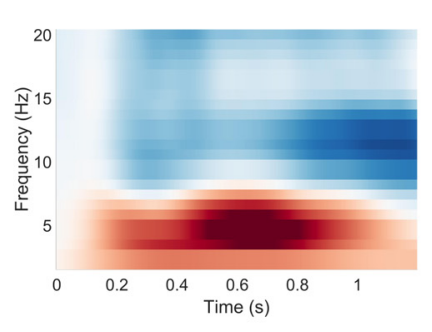

Both Conflicts

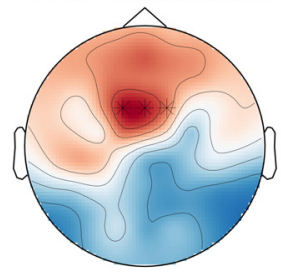
2

Figure 2. Condition-wise topographical plots and time-frequency heat maps. Topographical plots show average baseline-corrected power in the theta range (4-7 Hz) $0.4-0.8 \mathrm{~s}$ after stimulus onset. Heatmaps show average baseline-corrected oscillatory power over the whole frequency spectrum analyzed for the full trial interval at the electrodes $\mathrm{FC} / \mathrm{FC} 1 / \mathrm{FC} 2$ (marked with a star in the topographical plots) relative to stimulus onset.

Brain-behavior correlations. To investigate the relationship between neural activity and behavioral outcomes during conflict resolution, we computed correlation maps between midfrontal oscillatory activity and reaction time on the same trial in each condition (for similar approaches, see Cohen and Cavanagh, 2011; Cooper et al., 2019; Adelhöfer and Beste, 2020). More specifically, for each participant individually we calculated a separate time-frequency map with the same parameters as in the main analysis for each trial. For each participant, we then correlated every time-frequency point with the reaction time of the same trial separately for each condition. Both oscillatory activity and reaction times were rank transformed before the calculations of correlations, which lowers the potential influence of extreme values on correlation estimates (Conover, 2012; Cohen, 2014b). This analysis produced one time-frequency map of correlation coefficients per condition and participant. Thus, the average time-frequency maps of all participants represent the average correlation strength between oscillatory power and reaction times. We used cluster-based permutation with the same parameters as for the main power analysis, to test the correlation maps of all participants in each condition for clusters that differed from 0 . Correlation clusters that significantly deviate from 0 indicate significant correlations between oscillatory power and reaction times. We used cluster-based permutation with the same parameters as for the main power analysis to test the correlation maps of all participants in each condition for clusters which differed from 0 . Correlation clusters that significantly deviate from 0 indicate significant correlations between oscillatory power and reaction times.

Connectivity analysis. We calculated phase synchronization between the midfrontal electrodes selected for the main analysis (FCz/FC1/FC2) and all other electrodes as a measure of interconnectivity between the midfrontal and other brain regions. For estimating synchronicity, we used the debiased weighted phase lag index (WPLI; Vinck et al., 2011). Compared with other measures of synchronization, the weighted phase lag index is less susceptible to the influence of volume conduction, which can artificially inflate connectivity estimates (Bastos and Schoffelen, 2016). WPLI between the midfrontal seed electrodes and all other sensors was calculated separately for each condition. WPLI values for each seed electrode itself were set to 0 . Resulting synchronization values were baseline corrected by subtracting the mean WPLI between 0.30 and 0.10 s before trial onset. Thus, higher WPLI values at any electrode indicate an increase in synchronization between that sensor and the midfrontal areas. Synchronization values were averaged in $0.2 \mathrm{~s}$ intervals. Since we wanted to explore potential changes of connectivity between the midfrontal side and all other cortical regions, we used cluster-based permutation that included all electrodes and time points to identify spatiotemporal clusters of condition differences in synchronization in the theta range $(4-7 \mathrm{~Hz})$. To identify the electrodes with the most reliable effects, and to avoid false positives in this whole-head analysis, we chose a more conservative cutoff value for individual time-electrode points of $p<0.01$. The remaining analysis parameters were identical to the ones used for the main time-frequency analysis.

The whole-head analysis revealed a significant interaction between attentional and motor conflicts in the synchronization between midfrontal and parietal electrodes (see Fig. 7). To explore this interaction further, we calculated additional heat maps of the average midfrontal synchronicity for the parietal region, based on a representative array of the centroparietal area $(\mathrm{P} 1 / \mathrm{Pz} / \mathrm{P} 2 / \mathrm{PO} 3 / \mathrm{POz} / \mathrm{PO} 4)$. Permutation analysis with the same parameters as in the main analysis was used to test for effects of attentional and motor conflicts in this region.

Our whole-head analysis also revealed a pattern of lateralization in midfrontal-to-parietal synchronicity (see Fig. 7). Therefore, we chose to investigate the difference in contralateral and ipsilateral phase synchronization relative to the acting hand. For this purpose, we calculated the average synchronization values separately for the parietal contralateral side (electrodes CP5/CP3/CP1/P5/P3/P1) and the parietal ipsilateral side (electrodes $\mathrm{CP} 6 / \mathrm{CP} 4 / \mathrm{CP} 2 / \mathrm{P} 6 / \mathrm{P} 4 / \mathrm{P} 2$ ). Cluster-based permutation was used to compare the time-frequency maps of phase synchronization values between the contralateral and ipsilateral sides within each condition, as well as for comparing the difference in lateralization effect (contralateral-ipsilateral) between the different types of conflict.

Data availability. Data and materials of this study are archived online at https://osf.io/spwm9/?view_only=d9c0fd02768e4142bbcb110eaa2afc14.

\section{Results}

\section{Behavioral results}

Figure 3 shows box plots for error rates and reaction times. For error rates, we found a main effect of ATTENTION $\left(F_{(1,33)}=\right.$ $\left.85.65, p<0.001, \eta_{p}^{2}=0.72, \mathrm{BF}>10^{6}\right)$, a main effect of MOTOR $\left(F_{(1,33)}=64.60, p<0.001, \eta_{p}^{2}=0.66, \mathrm{BF}>10^{6}\right)$, as well as an ATTENTION * MOTOR interaction $\left(F_{(1,33)}=26.86, p<0.001\right.$, $\left.\eta_{p}^{2}=0.45, \mathrm{BF}=9.91\right)$. On average, the presence of a singleton distractor compared with search displays without singleton distractor increased the error rate by $5.81 \%(\mathrm{SD}, 4.48)$. Conflict actions compared with standard actions increased the error rate by $5.76 \%(\mathrm{SD}, 4.91)$. The increase in errors for conflict actions 
compared with standard actions was significantly higher when a singleton distractor was present compared with when no singleton distractor was shown $\left(t_{(33)}=5.18, p<0.001\right.$, $d=0.89, \mathrm{BF}=1936.56)$.

For reaction times, we found a main effect of $\operatorname{ATTENTION}\left(F_{(1,33)}=244.74, p<0.001, \eta_{p}^{2}=\right.$ $\left.0.88, \mathrm{BF}>10^{6}\right)$, a main effect of MOTOR $\left(F_{(1,33)}=325.42, p<0.001, \eta_{p}^{2}=0.91, \mathrm{BF}>10^{6}\right)$, but no ATTENTION * MOTOR interaction $\left(F_{(1,33)}<0.01, p=0.98, \mathrm{BF}=0.26\right)$. Trials with singleton distractors compared with trials without singletons increased reaction times by $60.1 \mathrm{~ms}$ (SD, 23.5). The need to perform conflict actions compared with standard actions increased reaction times by $80.8 \mathrm{~ms}$ ( $\mathrm{SD}, 27.0$ ). Overall, both the presence of singleton distractors and conflict actions interfered with participants' performance, as indicated by increases in both error rates and reaction times.

\section{Midfrontal oscillatory power}

Figure 2 shows time-frequency maps for midfrontal oscillatory power. Figure 4 shows the results of the cluster-based permutation for the ANOVA with the factors ATTENTION (no attentional conflict/attentional conflict) and MOTOR (no motor conflict/motor conflict). Tests of main effect and interactions were performed for both stimulus-centered data (relative to search display onset) and response-centered data (relative to motor response) to aid the distinction between oscillatory effects elicited by the sensory distractors and motor conflicts.

For the factor ATTENTION, we found a significant cluster within the theta range, as follows: stimulus centered: $0.64-1.2 \mathrm{~s}$, $p=0.001$; response centered: -0.05 to $0.4 \mathrm{~s}, p=0.035$. Trials with salient distractors showed higher theta power compared with trials without distractors, but only in the last half of the trials and only after the motor response. Thus, during task execution, the mere presence of a salient distractor did not lead to a significant increase in theta power. Additionally, the factor ATTENTION revealed a significant cluster in the alpha-tobeta range before motor response: response centered: -0.74 to $-0.3 \mathrm{~s}, p=0.043$. Compared with trials without distractors, trials with salient distractors showed lower alpha/beta power during response preparation.

For the factor MOTOR, we found a significant cluster, which indicated that trials with motor conflicts compared with trials without motor conflicts lead to higher power with a peak in the theta range, as follows: stimulus centered: $0.27-1.2 \mathrm{~s}, p<0.001$, response-centered: -0.8 to $0.08 \mathrm{~s}, p<0.001$. Increases in theta power for motor conflicts emerged $\sim 0.80 \mathrm{~s}$ before and peaked shortly before the motor reaction. Additionally, for the factor MOTOR we found a significant cluster in the alpha/beta range, indicating that motor conflicts compared with trials without motor conflicts led to significantly lower alpha/beta activity before the motor reaction (response centered: -0.8 to $-0.27 \mathrm{~s}$, $p=0.001$ ) as well as after the motor action (stimulus centered: 0.73-1.2 s, $p<0.001$; response centered: 0.09-0.4 s, $p=0.005$ ). Thus, motor conflicts led to increases in theta power, which were more most prominent directly before the motor response. Additionally, motor conflicts led to decreases in alpha/beta power.

Testing the ATTENTION * ACTION interaction revealed a significant cluster in the theta to alpha range in the stimulus- centered data $(0.44-0.77 \mathrm{~s}, p=0.045)$. There was no significant ATTENTION * MOTOR interaction in the response-centered data. As the interaction effect occurred during the latter part of the trial and was not significant in the response-centered analysis, the interaction in the stimulus-centered data could reflect differences in reaction times between the conditions. Thus, we found no consistent evidence for a midfrontal ATTENTION * MOTOR interaction.

In addition to the permutation ANOVA, we also present the direct comparisons of different types of conflicts via permutation $t$ tests in Figure 5. These tests confirmed the main findings of the ANOVA: compared with trials with only attentional conflicts, trials that contained either only a motor conflict or both motor and attentional conflicts simultaneously showed higher theta power in the later part of the trial before the motor response (all $p$ values $<0.002)$. Trials with attentional conflicts and motor conflicts did not differ in theta activity in the postresponse interval. Additionally, trials that contained only a motor conflict or both types of conflicts simultaneously compared with trials with only attentional conflicts showed lower alpha/beta power at the end of the trial (all $p$ values $<0.02$ ). The presence of both conflicts compared with only an attentional conflict also led to lower power in the alpha/beta range before the response $(p=0.002)$.

The simultaneous presence of distractor and motor conflicts compared with the presence of only a motor conflict showed a significantly higher power theta range at the end of the trial in the stimulus-centered data $(0.75-1.2 \mathrm{~s}, p=0.021)$ as well as a significantly lower power in the alpha range in the first half of the trial $(0.17-0.36 \mathrm{~s}, p=0.046)$. However, these effects did not occur in the response-centered data. Here, both conflicts simultaneously compared with trials with only motor conflicts showed a cluster of lower alpha/beta power during and after the motor response ( -0.08 to $0.26 \mathrm{~s}, p=0.034)$. This suggests that the significant clusters in the stimulus-centered comparison occurred mostly because of differences in the timing of neural activity. Thus, when controlled for differences in timing, both conflicts simultaneously compared with only motor conflicts did not lead to an increase in theta power, but only a decrease in alpha/beta power around and after the motor response.

To conclude, stimulus-locked analysis indicated increases in theta power for both attentional and motor conflicts, which were mostly confined to the second half of the trial. Importantly, for attentional conflicts, we found no evidence for early conflictrelated increases in theta power directly evoked by the onset of the distractor. Theta increases for attentional conflicts only occurred after the response, meaning after the conflict resolution 


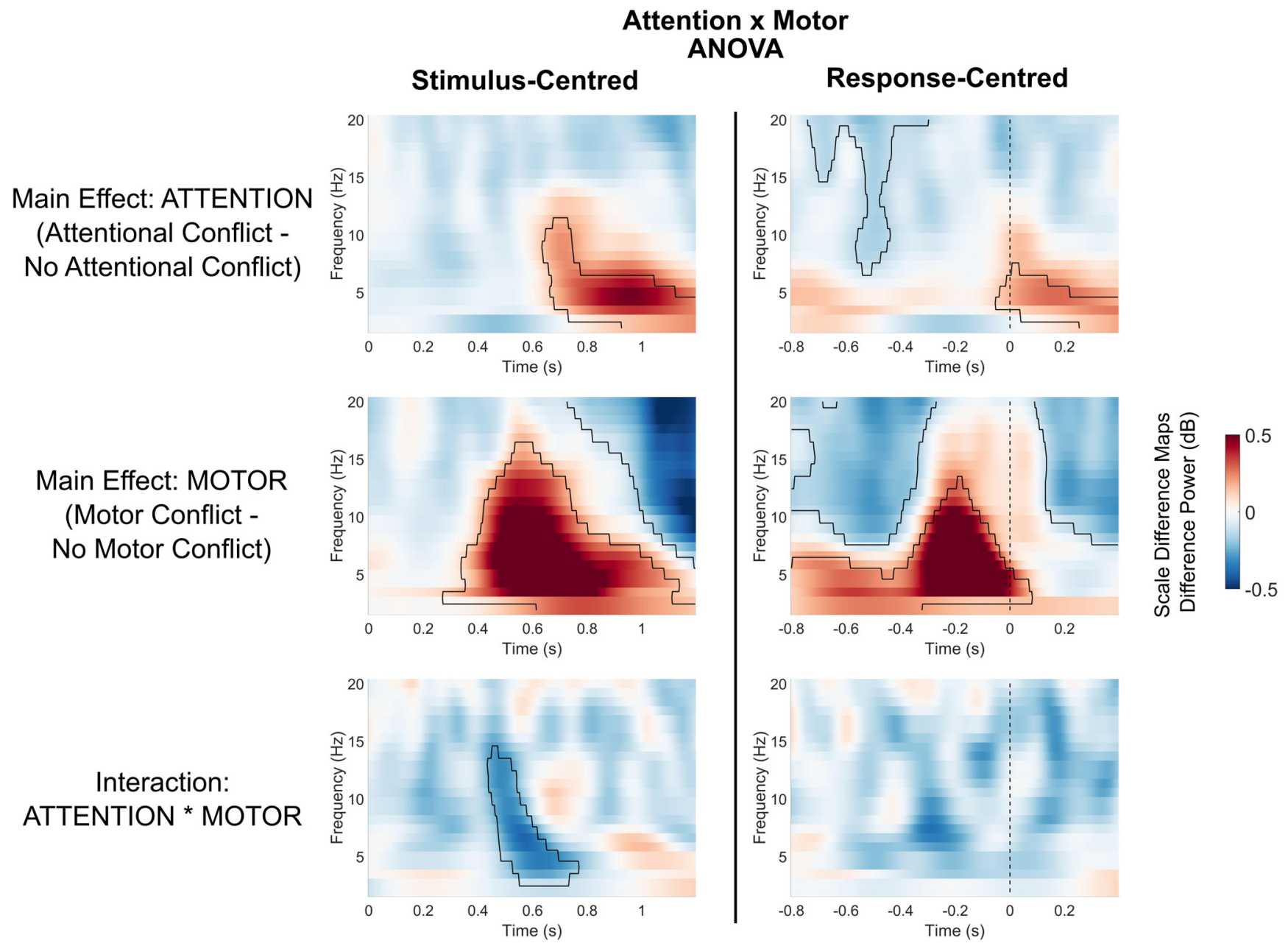

Figure 4. Main and interaction effects of attentional and motor conflicts on midfrontal power. Heatmaps show main and interaction effects on midfrontal power (averaged over electrodes $\mathrm{FC} / \mathrm{FC} 1 / \mathrm{FC}$ ). Marked areas show clusters of significant differences based on permutation analysis $(p<0.05)$. The vertical dashed line for response-centerd data indicates the time of motor response.

had already taken place. Conversely, response-locked data showed early and prolonged increases in theta power for motor conflicts. Thus, motor conflicts, but not attentional conflicts, were marked by increases in theta power during task execution. Both types of conflicts evoked decreases in alpha/beta power.

\section{Brain-behavior correlations}

Figure 6 shows average correlations between trial-wise reaction times and midfrontal oscillatory power. Analysis of stimuluscentered data revealed clusters of positive correlations between theta power and response times in the later part of the trial for all conditions, as follows: no conflict: $0.32-1.2 \mathrm{~s}, p<0.001$; attentional conflict: $0.34-1.2 \mathrm{~s}, p<0.001$; motor conflict: $0.47-1.2 \mathrm{~s}$, $p<0.001$; both conflicts: $0.56-1.2 \mathrm{~s}, p<0.001$. For trials without conflict, as well as for trials with attentional conflicts, we also found clusters of negative correlations in the alpha/beta frequency range at the end of the trial period (no conflict: $0.6-1.2 \mathrm{~s}$, $p=0.003$; attentional conflict: $0.9-1.2 \mathrm{~s}, p=0.013$ ).

Note that correlations of stimulus-centered data with reaction times could also occur because of temporal differences in the onset and offset of task-related neural activity, rather than because of a systematic relationship between the strength of neural activity and response times.

Importantly, the analysis of response-centered data confirmed the presence of positive correlations between theta power and reaction times. In every condition, positive correlation clusters could be observed as early as $0.8 \mathrm{~s}$ before the action execution up until the end of the time region of interest (no conflict: -0.8 to $0.32 \mathrm{~s}, p<0.001$; attentional conflict/motor conflict/both conflicts: -0.8 to $0.4 \mathrm{~s}, p<0.001$ ). Additionally, in every condition we found a cluster of negative correlations between alpha/ beta power and reaction times, which peaked $\sim 0.50 \mathrm{~s}$ before response execution (no conflict: -0.8 to $-0.26 \mathrm{~s}, p=0.002$; attentional conflict: -0.78 to $-0.33 \mathrm{~s}, p=0.003$; motor conflict: -0.72 to $-0.41 \mathrm{~s}, p=0.005$; both conflicts: -0.77 to $-0.45 \mathrm{~s}, p=0.005$ ).

To conclude, for all conditions, and independent of the type of conflict present, higher midfrontal theta power and lower alpha/beta power was predictive of motor slowing.

\section{Midfrontal theta synchronization}

Figure 7 shows average synchronization between the midfrontal electrodes $\mathrm{FCz} / \mathrm{FC} 1 / \mathrm{FC} 2$ and all other electrodes. We again conducted permutation tests for the factors ATTENTION, MOTOR, and the ATTENTION * MOTOR interaction. For the factor ATTENTION, we found no significant clusters in the stimuluscentered data, indicating that no significant changes in midfrontal connectivity could be found relative to the distractor onset. For response-centered data, we found a parietal-to-posterior cluster with a decrease in synchronization $(-0.6$ to $-0.2 \mathrm{~s}$, 


\section{Contrasts Between Conflict Types}

Response-Centred
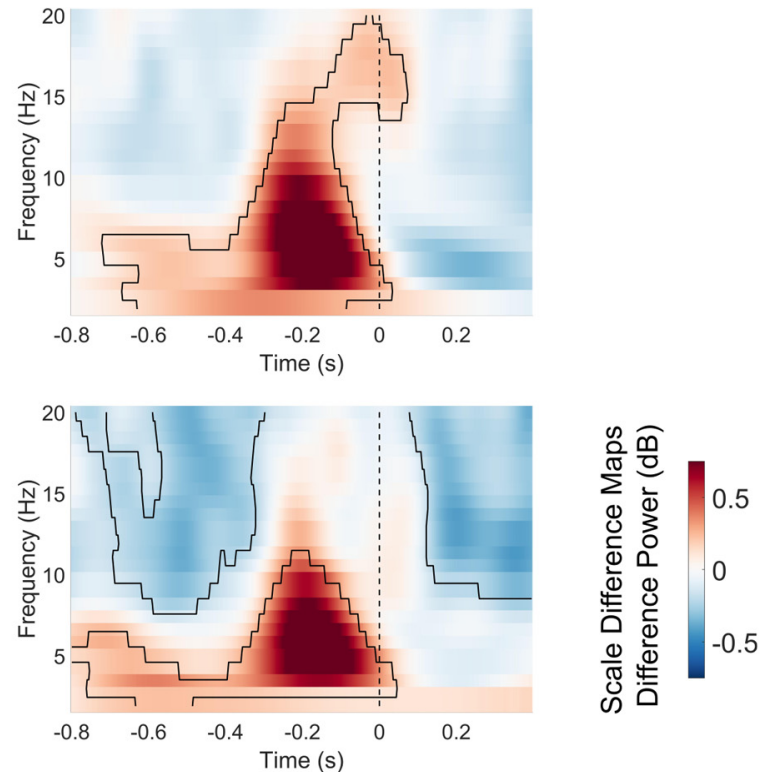

\section{Contrast: Both Conflicts - Motor Conflict}

\section{Contrast:} Attentional Conflict

\section{Contrast: Both Conflicts - Attentional Conflict}

\section{Stimulus-Centred}

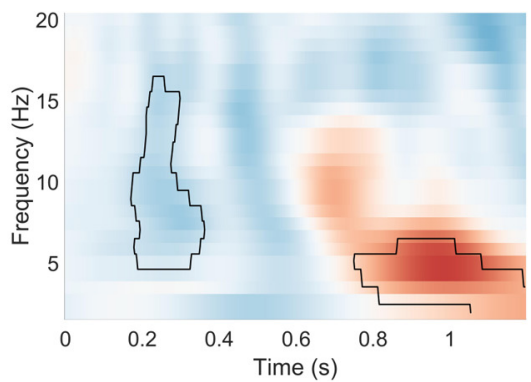

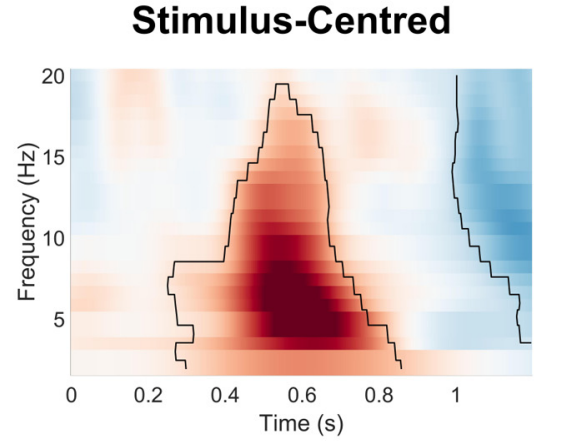
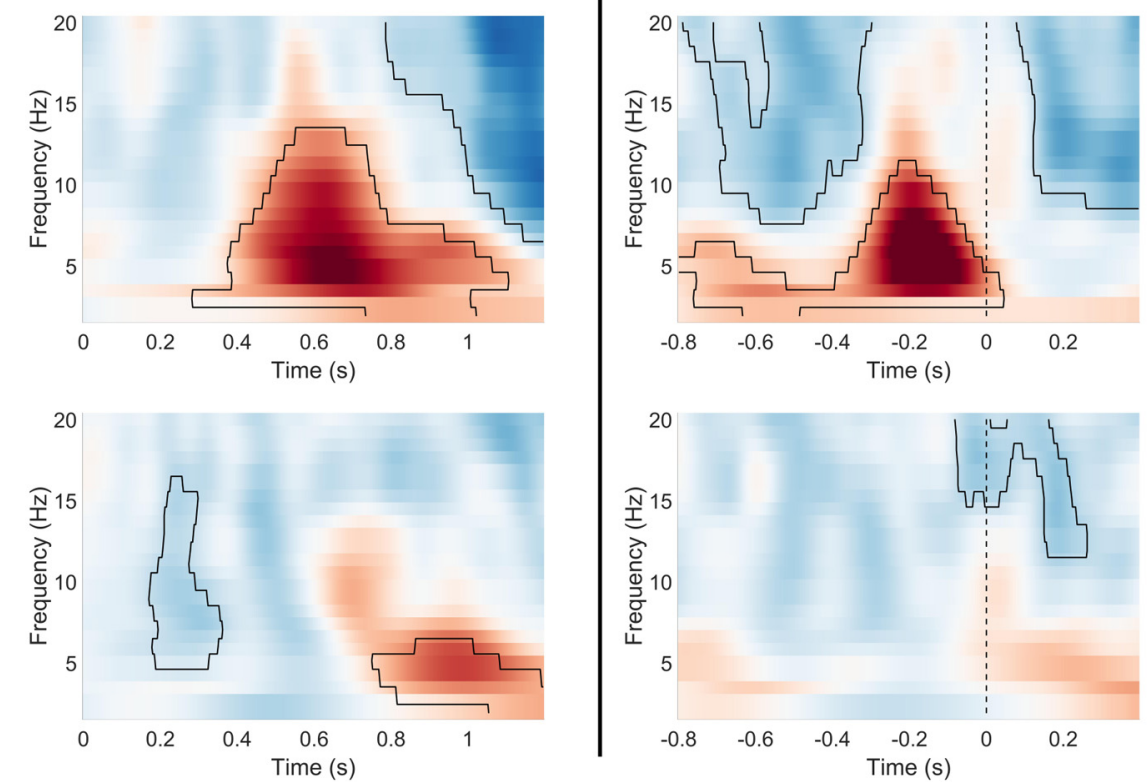

Figure 5. Condition-wise contrasts in oscillatory power between different conflict types. Heatmaps show differences in midfrontal power (averaged over electrodes $\mathrm{FCZ} / \mathrm{FC} 1 / \mathrm{FC} 2$ ). Marked areas show clusters of significant differences based on permutation analysis $(p<0.05)$. The vertical dashed line for response-centered data indicates the time of motor response.

$p<0.001)$. This suggests desynchronization between central and parietal electrodes during attentional conflicts.

For the factor MOTOR, stimulus-centered analysis indicated two significant clusters of synchronicity increases. These clusters comprised a wide array of electrodes, most of which were located at central and left parietal regions $(0.4-1 \mathrm{~s}, p<0.001)$ as well as the frontal region (0.4-1 s, $p=0.001)$. Analysis of response-centered data confirmed that the majority of significant increases occurred directly before the motor response $(-0.6$ to $0.2 \mathrm{~s}$, $p<0.001)$. Additionally, response-centered analysis indicated an early increase in synchronization with mostly left parietal electrodes $(-0.8$ to $-0.6 \mathrm{~s}, p=0.001)$. Thus, motor conflicts led to an early increase in synchronization with parietal electrodes, as well as a later increase in frontocentral connectivity around the time of response execution, including a noticeable peak in synchronization with left parietal electrodes. Since all motor actions in this experiment had to be performed with the right hand, this could indicate synchronization between midfrontal electrodes and parts of the parietal motor cortex contralateral to the acting hand.

We found evidence for an ATTENTION $*$ MOTOR interaction in centroparietal electrodes (stimulus centered: 0.4$0.6 \mathrm{~s}, p=0.030$; response centered: -0.6 to $-0.4 \mathrm{~s}, p=0.044$ ). This indicates that frontoparietal synchronization differed between trials where both conflicts were present compared with trials with either only attentional or motor conflicts. We explored this interaction effect in a separate analysis (see next section).

We also tested for differences in theta connectivity between the different types of conflicts via permutation $t$ tests. Figure 8 presents the results of these contrasts for response-centered data, since the main ANOVA showed that most significant changes in connectivity were locked to the motor response. The analogous analysis of the same contrasts for stimulus-centered data did not reveal any additional findings. As can be seen from Figure 8, contrasts between conflicts largely confirm the findings from the main analysis. Conditions that contained a motor conflict (motor conflict only or both attentional and motor conflict simultaneously) compared with trials with only attentional conflicts showed increases in theta synchronization over a wide array of electrodes, including a prominent increase on frontal and parietal electrodes (all $p$ values $<0.01$ ). Conversely, conditions that contained an attentional conflict compared with conditions that only contained motor conflicts showed significant decreases in synchronization mostly confined to parietal and posterior electrodes (all $p$ values $<0.03$ ).

To conclude, motor conflicts, but not attentional conflicts, led to an increase in theta synchronization with midfrontal electrodes across the cortex, including frontal and contralateral-parietal regions. In contrast, attentional conflicts showed a decrease in 


\section{Stimulus-Centred}
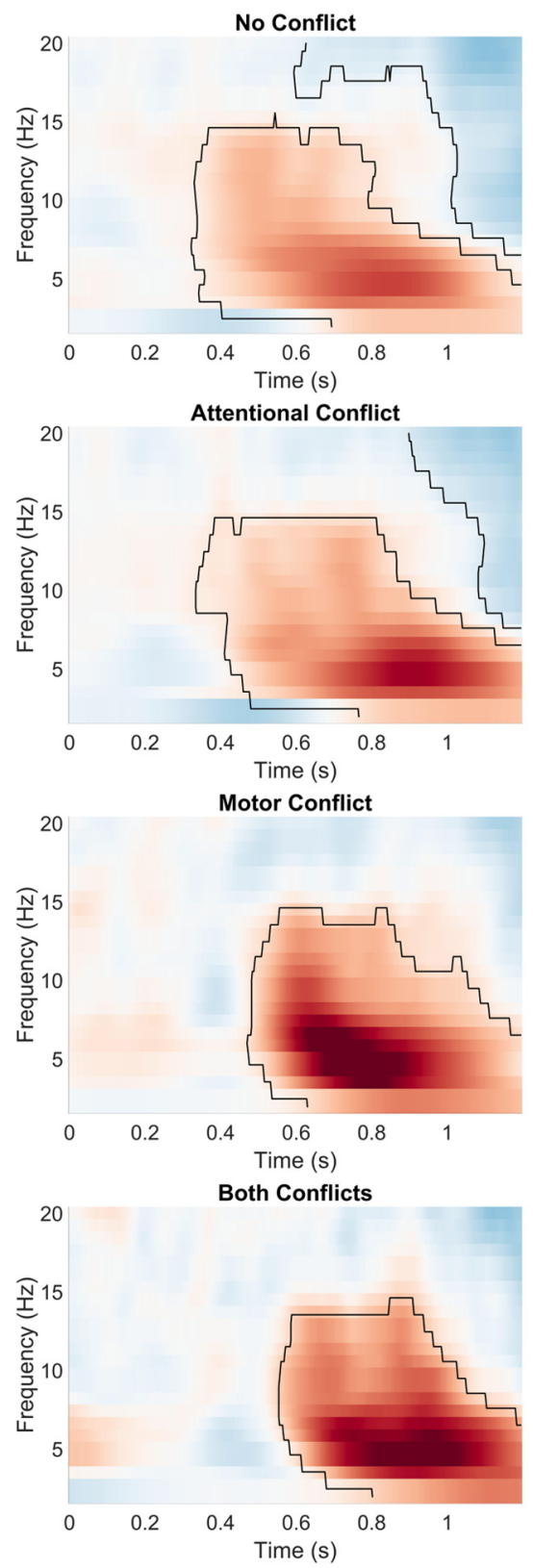

Response-Centred
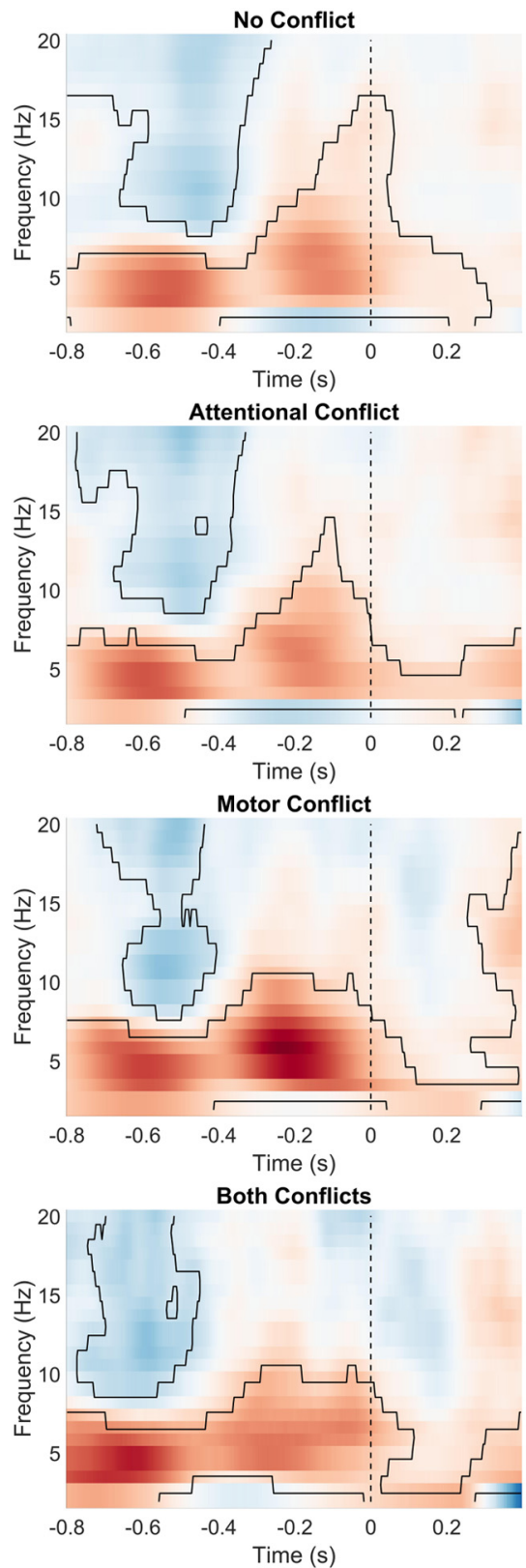

\section{Correlation Coefficient}

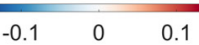

Figure 6. Correlations between midfrontal oscillatory power and reaction times. Heatmaps show correlations between reaction times and midfrontal oscillatory power (averaged over electrodes $F(z / F C 1 / F(2)$. Marked areas show clusters of significant correlations based on permutation analysis $(p<0.05)$. Vertical dashed line for response-centered data indicates the time of motor response.

midfrontal synchronization with centroparietal-to-posterior electrodes.

\section{Interaction effects in frontoparietal theta synchronization}

The whole-head connectivity analysis indicated an interaction effect between attentional and motor conflicts on centroparietal electrodes. To explore this interaction, we calculated average time-frequency maps of synchronicity between midfrontal and centroparietal electrodes, and again performed ATTENTION * MOTOR ANOVA on the resulting values (Fig. 9). For the factor ATTENTION, centroparietal electrodes showed a significant decrease in synchronization in the theta to alpha range $(-0.48$ to $-0.04 \mathrm{~s}, p<0.001)$. For the factor MOTOR, we also found a cluster of lower synchronization $(-0.53$ to $-0.3 \mathrm{~s}, p<0.001)$. Additionally, the factor MOTOR showed two clusters of increases in theta synchronization $(-0.8$ to $-0.42 \mathrm{~s}, p=0.001$; -0.23 to $0.08 \mathrm{~s}, p=0.006$ ).

As in the whole-head analysis, we found a significant ATTENTION $*$ MOTOR interaction in the theta range $(-0.62$ to $-0.39 \mathrm{~s}, p=0.031)$. We explored this interaction by testing the effect of motor conflicts separately for trials with and without attentional conflicts. For motor conflicts in the absence of salient 


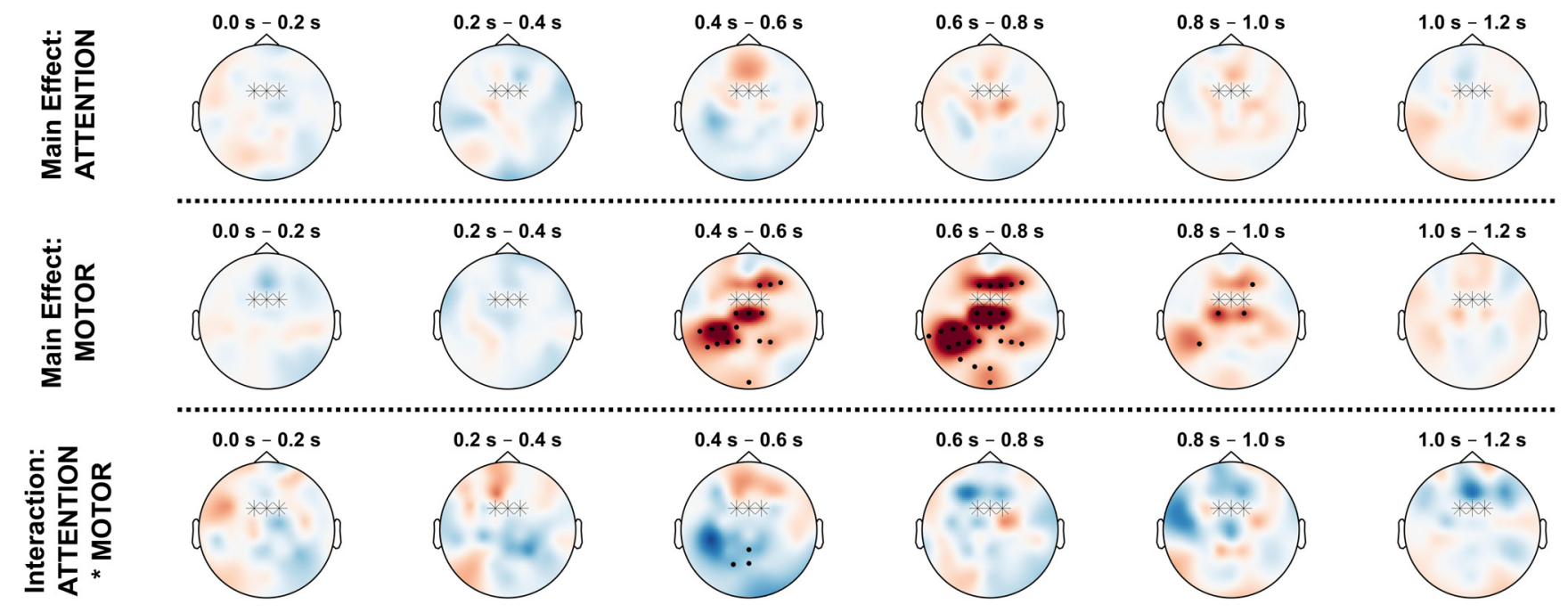

\section{Stimulus-Centred}

Response-Centred
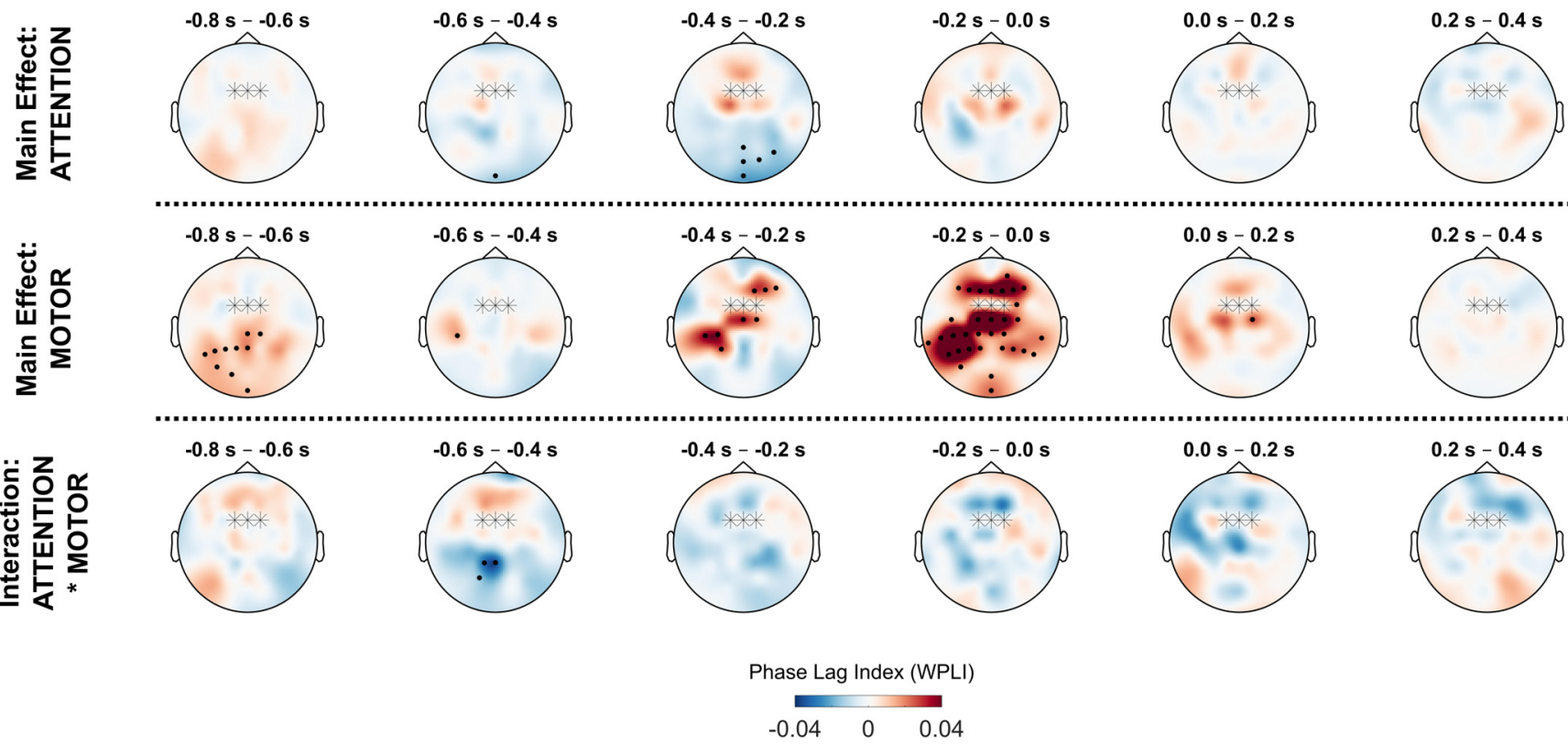

Figure 7. Conflict effects on theta synchronization with midfrontal electrodes. Topographical plots show average theta phase synchronization $(4-7 \mathrm{~Hz})$ between $\mathrm{FCz} / \mathrm{FC} 1 / \mathrm{FC} 2$ electrodes (marked with stars) and all other electrodes. Values for $\mathrm{FCz}$ are set to 0 . Black dots indicate electrodes belonging to significant clusters $(p<0.05)$.

distractors, we again found significant increases in theta synchronization $(-0.8$ to $-0.44 \mathrm{~s}, p<0.001 ;-0.25$ to $0.05 \mathrm{~s}, p=0.006)$. For motor conflicts on trials with salient distractors, however, there was no significant increase in theta synchronicity. Instead, we found a decrease in synchronization in the theta and alpha/beta range $(-0.53$ to $-0.32 \mathrm{~s}, p<0.001)$. To conclude, as indicated by the whole-head analysis, motor conflicts and attentional conflicts differed in their synchronization between midfrontal and centroparietal electrodes. While motor conflicts led to an increase in theta synchronization, attentional conflicts were marked by a decrease in synchronization in the theta and alpha ranges. As indicated by the interaction effect, the presence of sensory distractors led to lower theta synchronization during motor conflicts.

\section{Lateralization of theta synchronicity}

Based on the findings of the whole-head connectivity analysis, we further investigated the pattern of increased parietal synchronization contralateral to the acting hand in action conflicts. Figure 10 shows the comparison of contralateral-parietal and ipsilateral-parietal connectivity with the midfrontal region. For every condition, midfrontal phase synchronization in the delta/theta frequency range was stronger for the contralateral compared with the ipsilateral side around the time of the motor response (no conflict: -0.43 to $0.4 \mathrm{~s}, p=0.004$; attentional conflict: -0.25 to $0.4 \mathrm{~s}, p=0.005$; motor conflict: -0.34 to $0.32 \mathrm{~s}$, $p<0.001$; both conflicts: -0.36 to 0.09 s, $p=0.007$ ). We tested whether the lateralized difference in phase synchronicity (contralateral-ipsilateral) depended on the type of conflict using again an ATTENTION * MOTOR ANOVA. There was no main effect of ATTENTION, but a main effect of MOTOR showing an increase in lateralization $(-0.34$ to $0.04 \mathrm{~s}, p=0.001)$. There was no significant ATTENTION * MOTOR interaction. Thus, motor conflicts, but not attentional conflicts, increased the degree of lateralization. Individual condition contrasts confirmed that 

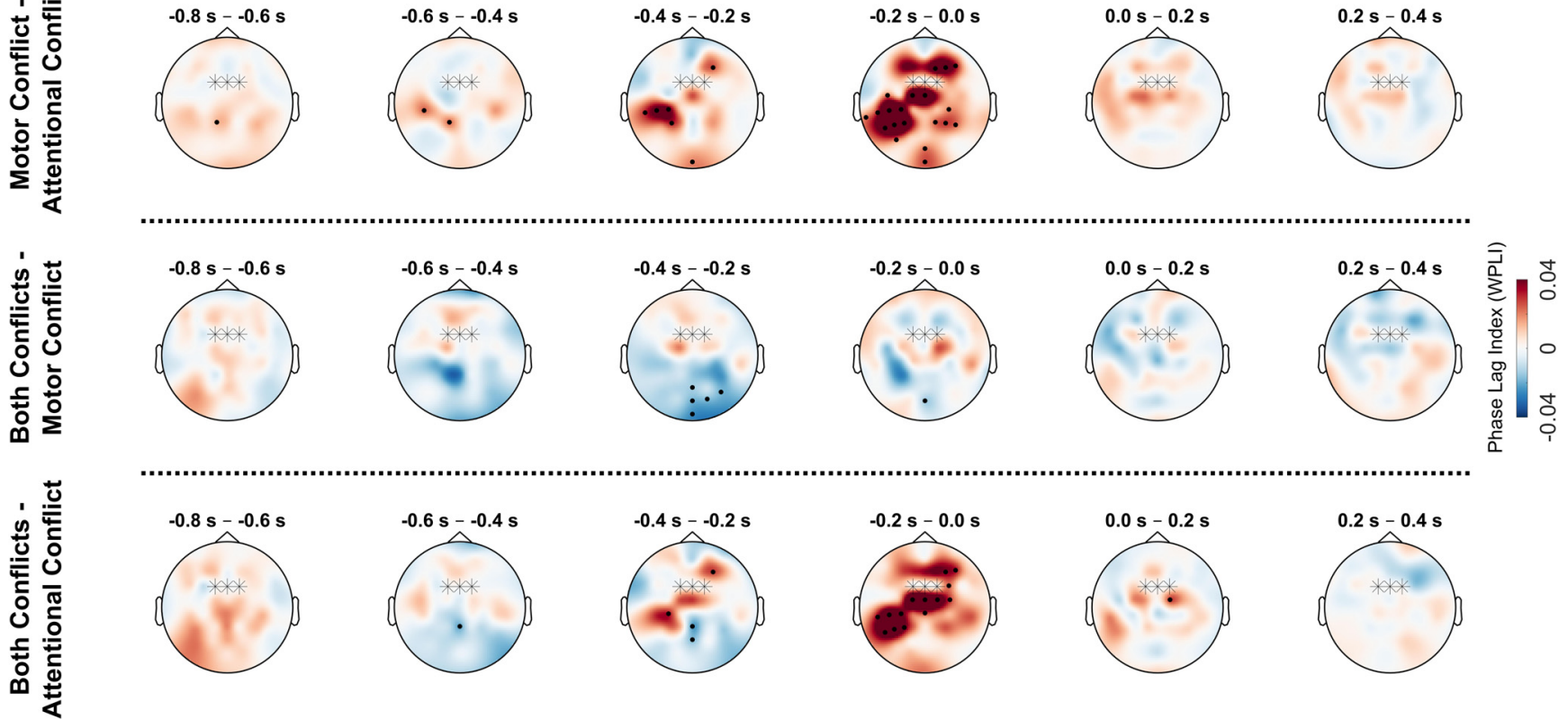

Figure 8. Condition-wise contrasts in theta syncronization between different conflict types. Topographical plots show average theta phase synchronization (4-7 Hz) between FCz/FC1/FC2 electrodes (marked with stars) and all other electrodes for response-centered data (with $0 \mathrm{~s}=$ motor response). Values for $\mathrm{FCZ} / \mathrm{FC1} / \mathrm{FC2}$ are set to 0 . Black dots indicate electrodes belonging to significant clusters $(p<0.05)$.

lateralization in synchronicity was significantly higher for motor conflict trials compared with trials with no conflict $(-0.3$ to 0.11 $s, p=0.002)$ as well as compared with trials with attentional conflicts $(-0.31$ to $0.11 \mathrm{~s}, p=0.001)$. There were no significant differences between trials with both attentional and motor conflicts compared with trials with only motor conflicts. To conclude, synchronization between the midfrontal and parietal regions was stronger contralateral to, rather than ipsilateral to, the acting hand in every condition. This lateralization of midfrontal synchronicity was most pronounced during motor conflicts.

\section{Discussion}

The current study compared midfrontal oscillatory dynamics between attentional conflicts, evoked via salient distractors, and motor conflicts, evoked via deviations from prepotent actions. While previous studies of cognitive control mostly tested the additive effects of sensory and motor interference, our design allowed for an independent manipulation of attentional and motor conflicts. Motor conflicts led to a clear spike in theta activity directly before the motor action when motor adjustments were most likely to take place. Conversely, for attentional conflicts there was no increase in theta power during conflict resolution. Moreover, while motor conflicts led to increased theta synchronization with the midfrontal region across the cortex, attentional conflicts led to a decrease in synchronization between midfrontal and parietal electrodes.

If the resolution of sensory interference was related to midfrontal theta oscillations, stimulus-locked analysis should have shown increases in theta activity directly after the distractor onset. Instead, as demonstrated by the response-locked analysis, for attentional conflicts we only found evidence for theta power increases after the conflict response had already been performed. Postresponse theta activity did not significantly differ between attentional and motor conflicts. Theta power increases after trials have been associated with proactive control processes, such as anticipatory adjustments for upcoming trials (van Driel et al.,
2012; Luft et al., 2013; Kaiser et al., 2020). Thus, our results would be compatible with the assumption that proactive control adjustments elicit theta power increases independent of the type of conflict (Cooper et al., 2015; Derosiere et al., 2018). Future studies could test this assumption directly by comparing theta power during pretrial intervals in which participants anticipate either sensory or motor interference. Importantly, our study shows that theta activity during the ongoing resolution of control conflicts is specific to motor conflicts and does not occur for sensory interference.

Decreases in alpha power are often associated with an increase in selective attention (Foster and Awh, 2019; van Diepen, et al., 2019). In the current study, both attentional and motor conflicts led to decreases in alpha/beta activity. This finding is consistent with previous studies that found both motor and sensory interference evoke alpha/beta suppression (McDermott et al., 2017; Popov et al., 2018). Thus, our results show that while an increase in midfrontal theta power oscillations during behavioral conflicts are specific to motor interference, decreases in the alpha/beta range occur independent of the source of interference.

In line with previous studies, we found that increases in theta power, as well as decreases in alpha power, were associated with slower responses in all conditions (Töllner et al., 2017). This finding suggests that midfrontal oscillatory activity during behavioral tasks partly reflects a speed-accuracy trade-off. Delaying immediate responses is assumed to be necessary to allow for the preparation of alternative behavior during demanding situations (Wessel and Aron, 2013; Logan et al., 2014; Elchlepp et al., 2016; Schall et al., 2017). Importantly, our results show that neither sensory nor motor interference fundamentally alters the functional relationship between midfrontal oscillations and motor responses.

Oscillatory synchronization is assumed to aid information exchange between task-relevant brain regions, which is an important aspect of neural conflict resolution (Fries, 2005; Cooper 

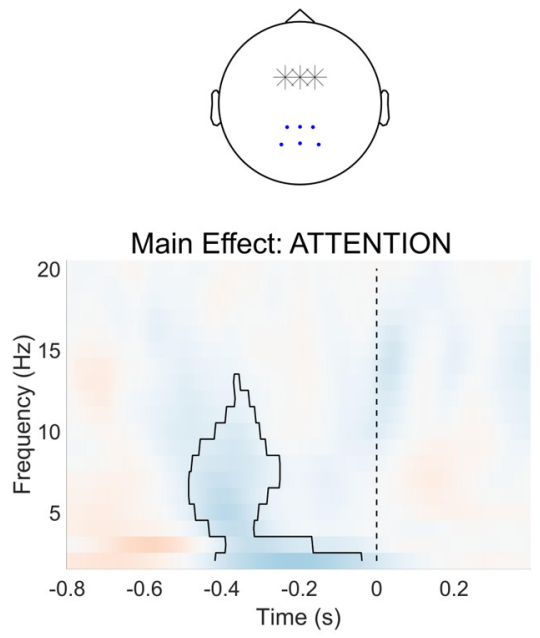

\section{ANOVA: ATTENTION * MOTOR}
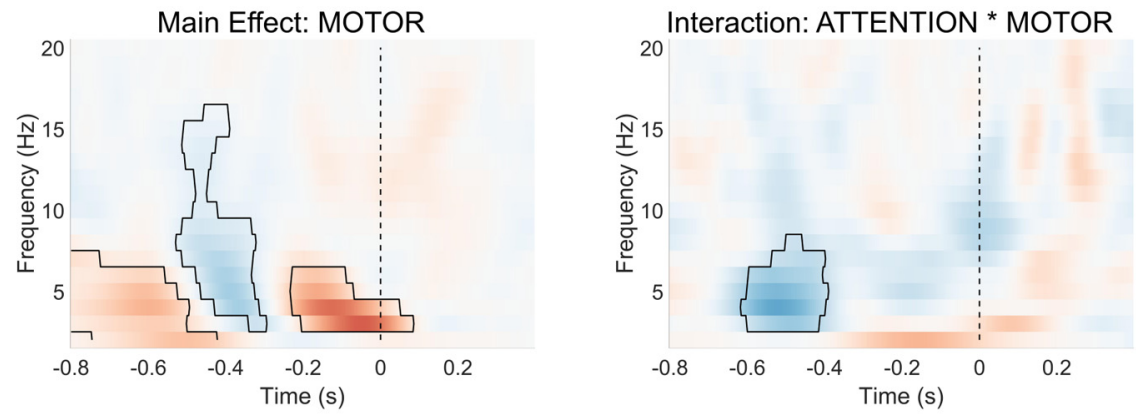

\section{Interaction Follow-Up: Post Hoc Comparison}

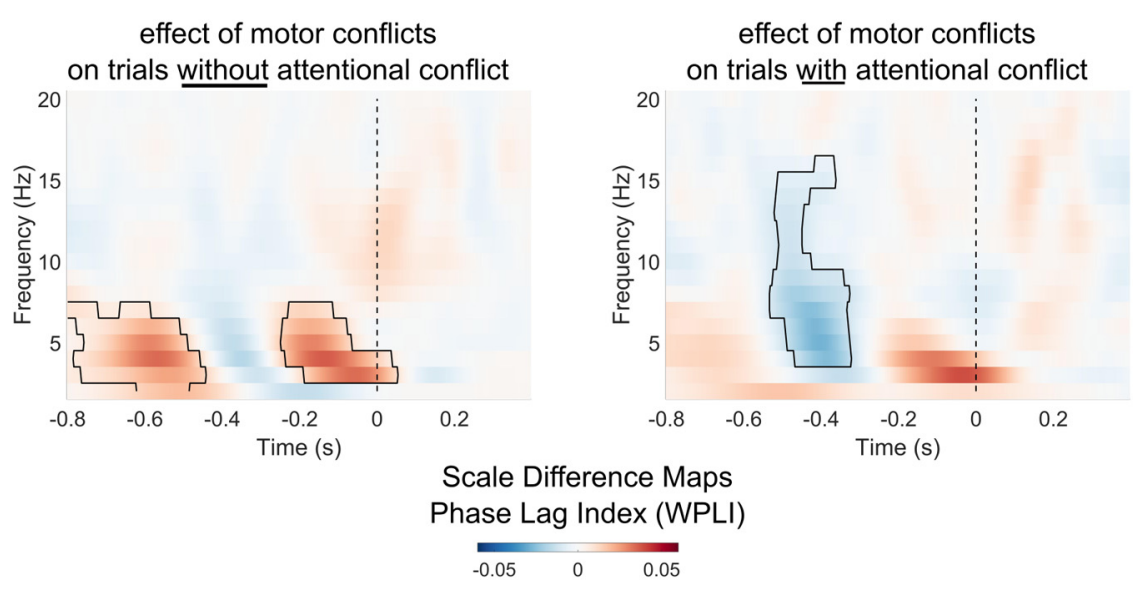

Figure 9. Syncronization between midfrontal and centroparietal electrodes. Heatmaps show synchronization between midfrontal electrodes (highlighted with stars in the topographical plot) and centroparietal electrodes (marked with dots). Marked areas in heat maps show clusters of significant effects based on permutation analysis $(p<0.05)$. The vertical dashed line indicates the time of motor response.

et al., 2015; van de Vijver et al., 2018; Duprez et al., 2019). One important question is in how far changes in synchronization are domain specific regarding the type of conflict affordance (van Driel et al., 2012; Vissers et al., 2018). This study provides important new evidence for the conflict-specific modulation of interbrain connectivity. Motor conflicts, but not attentional conflicts, led to markedly stronger theta synchronization around the time of response execution in a wide array of electrodes. This included significant lateralization in synchronicity in the left parietal region contralateral to the acting hand. This finding could indicate increased intercommunication between midfrontal regions and parts of the motor cortex related to the currently action-relevant effector. Thus, increases in theta synchronization were specific to the motor conflict that needed to be resolved.

In contrast to motor conflicts, attentional conflicts led to a decrease in synchronization between midfrontal and centroparietal electrodes to posterior electrodes, which occurred both in the theta and alpha range. Frontoparietal intercommunication has previously been associated with attentional processes (Corbetta and Shulman, 2002; Ptak, 2012; Marshall et al., 2015; Dixon et al., 2018). Additionally, cortical activity in posterior brain region is commonly associated with the processing of visual stimuli (Derosiere et al., 2018; Pastötter and Frings, 2018). Thus, the current findings suggest that visual distractors temporarily lower the intercommunication between the midfrontal regions and areas related to visual and attentional processing. Note that measures of phase synchronization are nondirectional and, thus, do not distinguish between the upstream (posterior-to-frontal) and downstream (frontal-toposterior) flow of information (Bastos and Schoffelen, 2016). On the one hand, this finding could be seen as evidence for neural distractor interference, which appears to lead to a temporary disruption in neural intercommunication. On the other hand, one could speculate that lowering the synchronization with parietal and posterior areas during salient distraction might be adaptive, since it could effectively lower the upstream propagation of distracting, task-irrelevant information. Independent of the functional relevance of distractorrelated desynchronization, this finding clearly demonstrates that motor conflicts, but not attentional conflicts, increase brainwide theta synchronization.

For interpreting the current findings, it is important to consider the feasibility of dissociating attentional and motor 

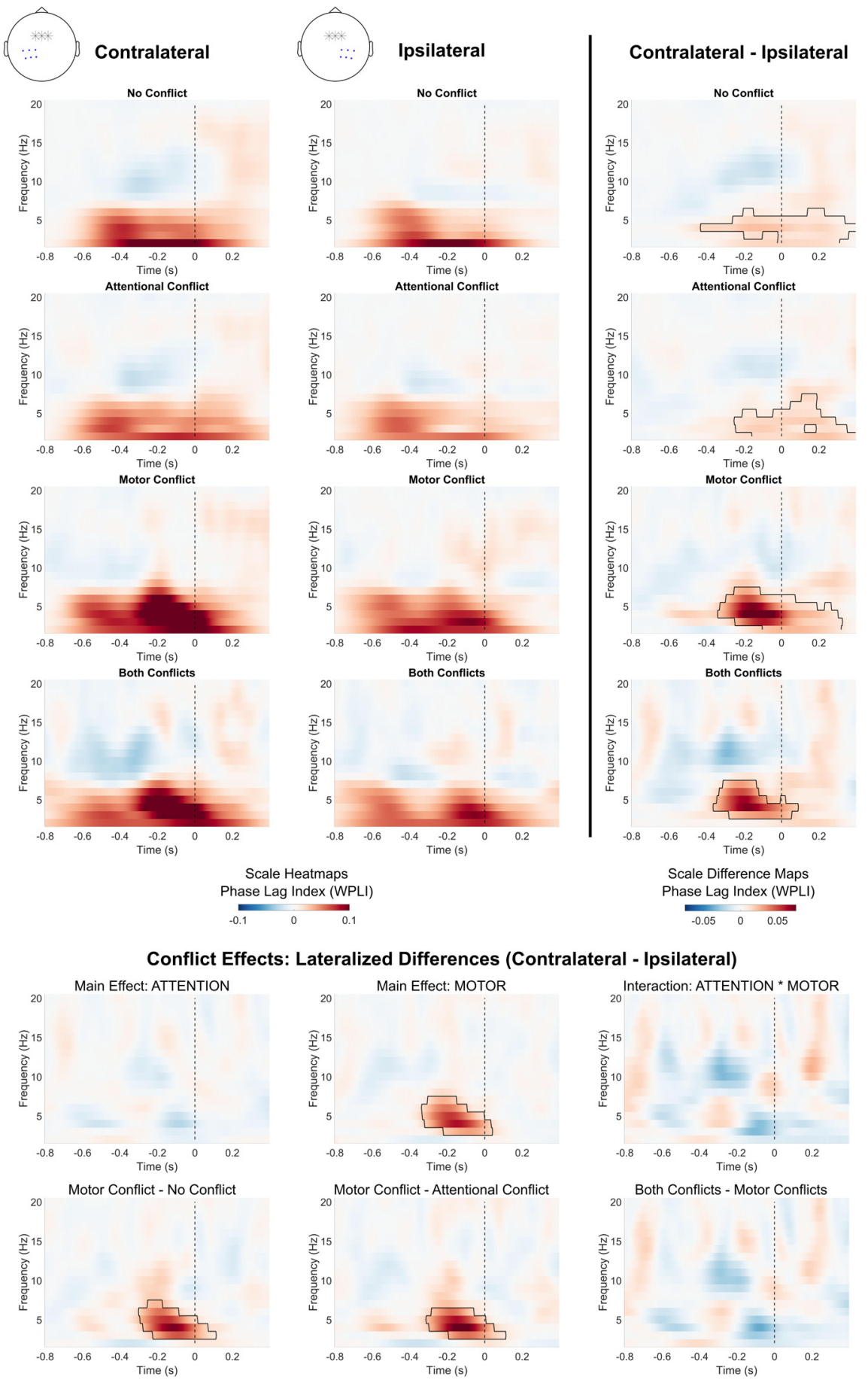

Figure 10. Lateralization of midfrontal theta synchronization. Blue dots in topographical plots show selected electrodes for heatmaps. Marked areas show clusters of significant differences based on permutation analysis $(p<0.05)$. The vertical dashed line indicates the time of motor response.

processes during conflict processing. In almost all behavioral tasks, including the current one, sensory and motor interference are, to a certain extent, intertwined. Since sensory distractors attract attention, suppressing distractors can entail suppression of automatic eye movements toward the distractor location. Thus, attentional conflicts can increase the need for adaptive motor control (Gaspelin and Luck, 2018b; Chelazzi et al., 2019). Conversely, motor conflicts most likely necessitate a speedy redirection of attentional resources to ensure the implementation of the correct motor response.
Thus, motor conflicts can likewise increase the need for attentional control (Mackie et al., 2013; Elchlepp et al., 2016). The finding that both attentional and motor conflicts lead to decreases in alpha power is consistent with the view that both attentional and motor conflicts evoked increased attentional control. Accordingly, our experimental manipulation should not be viewed as a complete separation of attentional and motor interference. However, given the inherent interdependence of attentional and motor adjustments, it seems striking that our results do show clear differences between attentional and motor conflicts with respect to midfrontal theta power and connectivity. This suggests that the current approach allows for at least a partial dissociation of motor-specific and attention-specific midfrontal control processes beyond what was previously described in the literature.

The present study focused on reactive control during sensory and motor interference. Accordingly, our results cannot rule out that increases in midfrontal theta power can also occur in other behavioral task contexts (Hsieh and Ranganath, 2014; Kaiser et al., 2021; Riddle et al., 2020). Recent studies have found evidence that different neural generators project theta activity on the midfrontal cortex (Töllner et al., 2017; Zuure et al., 2020). Thus, rather than a unitary neural mechanism, midfrontal theta activity could reflect different underlying neural processes depending on the task context.

With respect to the implementation of cognitive control, theta oscillations represent one of the most frequently discussed neural phenomena. Previous studies tended to conceptualize midfrontal theta oscillations as a "lingua franca" of cognitive control, meaning part of a general, domain-independent mechanism for conflict resolution (Cavanagh et al., 2012; Cavanagh and Frank, 2014; Duprez et al., 2019; Kaiser et al., 2019). In contrast, our results show that midfrontal theta oscillations during reactive control are not independent of the source of interference, but are more specific to motor-related adjustments. This represents a novel interpretation of midfrontal theta oscillations during reactive control, by demonstrating domain-specific effects of theta power and connectivity. The further dissociation between general and domain-specific neural effects during different types of behavioral interferences is an important step in understanding the neural implementation of cognitive control. 


\section{References}

Adelhöfer N, Beste C (2020) Pre-trial theta band activity in the ventromedial prefrontal cortex correlates with inhibition-related theta band activity in the right inferior frontal cortex. Neuroimage 219:117052.

Aron AR (2011) From reactive to proactive and selective control: developing a richer model for stopping inappropriate responses. Biol Psychiatry 69: e55-e68.

Bastos AM, Schoffelen J-M (2016) A tutorial review of functional connectivity analysis methods and their interpretational pitfalls. Front Syst Neurosci 9:175

Botvinick MM, Cohen JD, Carter CS (2004) Conflict monitoring and anterior cingulate cortex: an update. Trends Cogn Sci 8:539-546.

Cavanagh JF, Frank MJ (2014) Frontal theta as a mechanism for cognitive control. Trends Cogn Sci 18:414-421.

Cavanagh JF, Zambrano-Vazquez L, Allen JJB (2012) Theta lingua franca: a common mid-frontal substrate for action monitoring processes. Psychophysiology 49:220-238.

Chang A, Ide JS, Li H-H, Chen C-C, Li C-SR (2017) Proactive control: neural oscillatory correlates of conflict anticipation and response slowing. Eneuro 4:ENEURO.0061-17.2017.

Chelazzi L, Marini F, Pascucci D, Turatto M (2019) Getting rid of visual distractors: the why, when, how and where. Curr Opin Psychol 29:135-147.

Clayton MS, Yeung N, Cohen Kadosh R (2018) The many characters of visual alpha oscillations. Eur J Neurosci 48:2498-2508.

Cocchi L, Zalesky A, Fornito A, Mattingley JB (2013) Dynamic cooperation and competition between brain systems during cognitive control. Trends Cogn Sci 17:493-501.

Cohen MX (2014a) A neural microcircuit for cognitive conflict detection and signaling. Trends Neurosci 37:480-490.

Cohen MX (2014b) Analyzing neural time series data: theory and practice. Cambridge, MA: MIT.

Cohen MX (2015) Effects of time lag and frequency matching on phasebased connectivity. J Neurosci Methods 250:137-146.

Cohen MX, Cavanagh JF (2011) Single-trial regression elucidates the role of prefrontal theta oscillations in response conflict. Front Psychol 2:30.

Conover WJ (2012) The rank transformation-an easy and intuitive way to connect many nonparametric methods to their parametric counterparts for seamless teaching introductory statistics courses. WIREs Comp Stat 4:432-438.

Cooper PS, Wong ASW, Fulham WR, Thienel R, Mansfield E, Michie PT, Karayanidis F (2015) Theta frontoparietal connectivity associated with proactive and reactive cognitive control processes. Neuroimage 108:354363.

Cooper PS, Darriba Á, Karayanidis F, Barceló F (2016) Contextually sensitive power changes across multiple frequency bands underpin cognitive control. Neuroimage 132:499-511.

Cooper PS, Karayanidis F, McKewen M, McLellan-Hall S, Wong ASW, Skippen P, Cavanagh JF (2019) Frontal theta predicts specific cognitive control-induced behavioural changes beyond general reaction time slowing. Neuroimage 189:130-140.

Corbetta M, Shulman GL (2002) Control of goal-directed and stimulusdriven attention in the brain. Nat Rev Neurosci 3:201-215.

Criaud M, Boulinguez P (2013) Have we been asking the right questions when assessing response inhibition in go/no-go tasks with fMRI? A meta-analysis and critical review. Neurosci Biobehav Rev 37:11-23.

Derosiere G, Klein P-A, Nozaradan S, Zénon A, Mouraux A, Duque J (2018) Visuomotor correlates of conflict expectation in the context of motor decisions. J Neurosci 38:9486-9504.

Dixon ML, De La Vega A, Mills C, Andrews-Hanna J, Spreng RN, Cole MW, Christoff K (2018) Heterogeneity within the frontoparietal control network and its relationship to the default and dorsal attention networks. Proc Natl Acad Sci U S A 115:E1598-E1607.

Duprez J, Gulbinaite R, Cohen MX (2019) Midfrontal theta phase coordinates behaviorally relevant brain computations during response conflict. Neuroimage 207:116340.

Egner T (2008) Multiple conflict-driven control mechanisms in the human brain. Trends Cogn Sci 12:374-380.

Elchlepp H, Lavric A, Chambers CD, Verbruggen F (2016) Proactive inhibitory control: a general biasing account. Cogn Psychol 86:27-61.

Foster JJ, Awh E (2019) The role of alpha oscillations in spatial attention: limited evidence for a suppression account. Curr Opin Psychol 29:34-40.
Fries P (2005) A mechanism for cognitive dynamics: neuronal communication through neuronal coherence. Trends Cogn Sci 9:474-480.

Gaspar JM, McDonald JJ (2014) Suppression of salient objects prevents distraction in visual search. J Neurosci 34:5658-5666. 2014

Gaspelin N, Luck SJ (2018a) Combined electrophysiological and behavioral evidence for the suppression of salient distractors. J Cogn Neurosci 30:1265-1280.

Gaspelin N, Luck SJ (2018b) The role of inhibition in avoiding distraction by salient stimuli. Trends Cogn Sci 22:79-92.

Gratton G, Cooper P, Fabiani M, Carter CS, Karayanidis F (2018) Dynamics of cognitive control: theoretical bases, paradigms, and a view for the future. Psychophysiology 55:e13016.

Hampshire A, Sharp DJ (2015) Contrasting network and modular perspectives on inhibitory control. Trends Cogn Sci 19:445-452.

Harper J, Malone SM, Iacono WG (2017) Theta- and delta-band EEG network dynamics during a novelty oddball task. Psychophysiology 54:1590-1605

Hsieh LT, Ranganath C (2014) Frontal midline theta oscillations during working memory maintenance and episodic encoding and retrieval. Neuroimage 85:721-729.

Jarosz AF, Wiley J (2014) What are the odds? A practical guide to computing and reporting Bayes factors. J Probl Solving 7:2-9.

Kaiser J, Schütz-Bosbach S (2019) Proactive control without midfrontal control signals? The role of midfrontal oscillations in preparatory conflict adjustments. Biol Psychol 148:107747.

Kaiser J, Simon NA, Sauseng P, Schütz-Bosbach S (2019) Midfrontal neural dynamics distinguish between general control and inhibition-specific processes in the stopping of motor actions. Sci Rep 9:1-11.

Kaiser J, Belenya R, Chung W, Gentsch A, Schütz-Bosbach S (2021) Learning something new versus changing your ways: distinct effects on midfrontal oscillations and cardiac activity for learning and flexible adjustments. Neuroimage 226:117550.

Kerzel D, Barras C, Grubert A (2018) Suppression of salient stimuli inside the focus of attention. Biol Psychol 139:106-114.

Liesefeld HR, Liesefeld AM, Müller HJ (2019) Distractor-interference reduction is dimensionally constrained. Vis Cogn 27:247-259.

Logan GD, Van Zandt T, Verbruggen F, Wagenmakers EJ (2014) On the ability to inhibit thought and action: general and special theories of an act of control. Psychol Rev 121:66-95.

Luft CDB, Nolte G, Bhattacharya J (2013) High-learners present larger midfrontal theta power and connectivity in response to incorrect performance feedback. J Neurosci 33:2029-2038.

Mackie MA, Van Dam NT, Fan J (2013) Cognitive control and attentional functions. Brain Cogn 82:301-312.

Maris E, Oostenveld R (2007) Nonparametric statistical testing of EEG- and MEG-data. J Neurosci Methods 164:177-190.

Marshall TR, Bergmann TO, Jensen O (2015) Frontoparietal structural connectivity mediates the top-down control of neuronal synchronization associated with selective attention. PLoS Biol 13:e1002272.

McDermott TJ, Wiesman AI, Proskovec AL, Heinrichs-Graham E, Wilson TW (2017) Spatiotemporal oscillatory dynamics of visual selective attention during a flanker task. Neuroimage 156:277-285.

Mückschel M, Dippel G, Beste C (2017) Distinguishing stimulus and response codes in theta oscillations in prefrontal areas during inhibitory control of automated responses. Hum Brain Mapp 38:5681-5690.

Nigbur R, Ivanova G, Stürmer B (2011) Theta power as a marker for cognitive interference. Clin Neurophysiol 122:2185-2194.

Nigbur R, Cohen MX, Ridderinkhof KR, Stürmer B (2012) Theta dynamics reveal domain-specific control over stimulus and response conflict. J Cogn Neurosci 24:1264-1274.

Oostenveld R, Fries P, Maris E, Schoffelen JM (2011) FieldTrip: open source software for advanced analysis of MEG, EEG, and invasive electrophysiological data. Comput Intell Neurosci 2011:156869.

Pastötter B, Frings C (2018) It's the other way around! Early modulation of sensory distractor processing induced by late response conflict. J Cogn Neurosci 30:985-998.

Popov T, Westner BU, Silton RL, Sass SM, Spielberg JM, Rockstroh B, Heller W, Miller GA (2018) Time course of brain network reconfiguration supporting inhibitory control. J Neurosci 38:4348-4356.

Ptak R (2012) The frontoparietal attention network of the human brain: action, saliency, and a priority map of the environment. Neuroscientist 18:502-515. 
Riddle J, Vogelsang DA, Hwang K, Cellier D, D’Esposito M (2020) Distinct oscillatory dynamics underlie different components of hierarchical cognitive control. J Neurosci 40:4945-4953.

Sawaki R, Luck SJ (2010) Capture versus suppression of attention by salient singletons: electrophysiological evidence for an automatic attend-to-me signal. Atten Percept Psychophys 72:1455-1470.

Schall JD, Palmeri TJ, Logan GD (2017) Models of inhibitory control. Philos Trans R Soc Lond B Biol Sci 372:20160193.

Schwertman NC, Owens MA, Adnan R (2004) A simple more general boxplot method for identifying outliers. Comput Stat Data Anal 47:165-174.

Soutschek A, Taylor PCJ, Müller HJ, Schubert T (2013) Dissociable networks control conflict during perception and response selection: a transcranial magnetic stimulation study. J Neurosci 33:5647-5654.

Töllner T, Wang Y, Makeig S, Müller HJ, Jung T-P, Gramann K (2017) Two independent frontal midline theta oscillations during conflict detection and adaptation in a Simon-type manual reaching task. J Neurosci 37:2504-2515.

Van de Schoot R, Kaplan D, Denissen J, Asendorpf JB, Neyer FJ, van Aken MAG (2014) A gentle introduction to Bayesian analysis: applications to developmental research. Child Dev 85:842-860.

van de Vijver I, van Driel J, Hillebrand A, Cohen MX (2018) Interactions between frontal and posterior oscillatory dynamics support adjustment of stimulus processing during reinforcement learning. Neuroimage 181:170-181.

Van Diepen RM, Foxe JJ, Mazaheri A (2019) The functional role of alphaband activity in attentional processing: the current zeitgeist and future outlook. Curr Opin Psychol 29:229-238.

van Driel J, Ridderinkhof KR, Cohen MX (2012) Not all errors are alike: theta and alpha EEG dynamics relate to differences in error-processing dynamics. J Neurosci 32:16795-16806.
Verbruggen F, Notebaert W, Liefooghe B, Vandierendonck A (2006) Stimulus- and response-conflict-induced cognitive control in the flanker task. Psychon Bull Rev 13:328-333.

Vinck M, Oostenveld R, Van Wingerden M, Battaglia F, Pennartz CMA (2011) An improved index of phase-synchronization for electrophysiological data in the presence of volume-conduction, noise and sample-size bias. Neuroimage 55:1548-1565.

Vissers ME, Ridderinkhof KR, Cohen MX, Slagter HA (2018) Oscillatory mechanisms of response conflict elicited by color and motion direction: an individual differences approach. J Cogn Neurosci 30:468-481.

Wagenmakers E-J, Love J, Marsman M, Jamil T, Ly A, Verhagen J, Selker R, Gronau QF, Dropmann D, Boutin B, Meerhoff F, Knight P, Raj A, van Kesteren E-J, van Doorn J, Šmíra M, Epskamp S, Etz A, Matzke D, de Jong T, et al. (2018) Bayesian inference for psychology. Part II: example applications with JASP. Psychon Bull Rev 25:58-76.

Wessel JR (2018) Prepotent motor activity and inhibitory control demands in different variants of the go/no-go paradigm. Psychophysiology 55: e12871.

Wessel JR, Aron AR (2013) Unexpected events induce motor slowing via a brain mechanism for action-stopping with global suppressive effects. J Neurosci 33:18481-18491.

Wessel JR, Aron AR (2014) Inhibitory motor control based on complex stopping goals relies on the same brain network as simple stopping. Neuroimage 103:225-234.

Yamanaka K, Yamamoto Y (2010) Single-trial EEG power and phase dynamics associated with voluntary response inhibition. J Cogn Neurosci 22:714-727.

Zuure MB, Hinkley LB, Tiesinga PHE, Nagarajan SS, Cohen MX (2020) Multiple midfrontal thetas revealed by source separation of simultaneous MEG and EEG. J Neurosci 40:7702-7713. 